\title{
Anterior Sagittal Approach and Total Urogenital Mobilization for the Treatment of Persistent Urogenital Sinus in a 2-Year-Old Girl
}

\author{
Mario Lima ${ }^{1}$ Francesca Destro $^{1}$ Noemi Cantone ${ }^{1}$ Mohamed Mahmoud Abd El-Aleem Shalaby ${ }^{2}$ \\ Giovanni Ruggeri ${ }^{1}$
}

${ }^{1}$ Department of Pediatric Surgery, S. Orsola Malpighi Polyclinic, Bologna, Italy

2 Department of Pediatric Surgery, Tanta University Hospital, Egypt

Address for correspondence Mario Lima, PhD, Department of Pediatric Surgery, S. Orsola Malpighi Polyclinic, Bologna, Italy (e-mail: mario.lima@unibo.it).

Eur J Pediatr Surg Rep 2016;4:13-16.

\begin{abstract}
Persistent urogenital sinus (UCS) is a developmental anomaly. It represents one of the most complex problems that a pediatric surgeon may deal with. We report the case of a patient with UGS treated at 3 years of age by anterior sagittal transrectal approach and

Keywords

- urogenital sinus

- pediatric surgery

- total urogenital mobilization en bloc sinus mobilization. The procedure was performed with the patient prone with the initial idea of performing an anterior sagittal transrectal approach. The described approach allows an excellent anatomical view with a midline muscle sparing incision, along with an easy identification of the vaginal confluence with the benefit of avoiding dissection between the urethra and vagina.
\end{abstract}

\section{Importance for a Pediatric Surgeon}

Our proposed approach for UGS, combines the advantages of ASTRA and TUM. It is an anterior sagittal approach that does not require rectum splitting with the benefit of avoiding dissection between the urethra and vagina. It is a feasible procedure, which provides excellent exposure and easy identification of the vaginal confluence for congenital adrenal hyperplasia associated with long UGS.

\section{Introduction}

Persistent urogenital sinus (UGS) is a complex, challenging, and controversial pathology for the pediatric surgeon. It consists of a persistent communication of the genital and urinary tract with the urethra and vagina joined in a single common channel (CC), with the UGS reaching the perineum. ${ }^{1}$ It can be an isolated anomaly or associated with external genitalia virilization (congenital adrenal hyperplasia [CAH]), or included in the cloacal anomaly. ${ }^{2-4}$ Surgery should be tailored to the individual patient according to either the length of the CC (that identifies low and high forms) or to the associated anomalies. Simple flap vaginoplasties are sufficient to treat low UGS, whereas high forms require a more extensive dissection and a subsequent pull-through. ${ }^{3,4}$ This can be obtained by prior separation of the vagina and urethra (by the posterior sagittal transanorectal approach [PSTA] or the anterior sagittal transrectal approach [ASTRA]) or by an en bloc procedure (total urogenital mobilization [TUM]). What we are reporting in this article is a modified technique combining the advantages of these two approaches which was performed on a patient with high UGS related to $\mathrm{CAH}$. The aim of opting for this combined approach was to reduce surgical trauma and to provide optimum anatomical visualization, thus simplifying surgery and obtaining good functional and cosmetic results. received

April 16, 2015

accepted after revision

February 25, 2016

published online

April 5, 2016

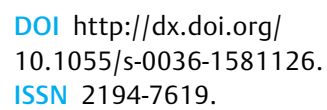

DOI http://dx.doi.org/ 10.1055/s-0036-1581126. ISSN 2194-7619.

(c) 2016 Georg Thieme Verlag KG
Stuttgart · New York

License terms

(1) $\circledast \circledast$ 


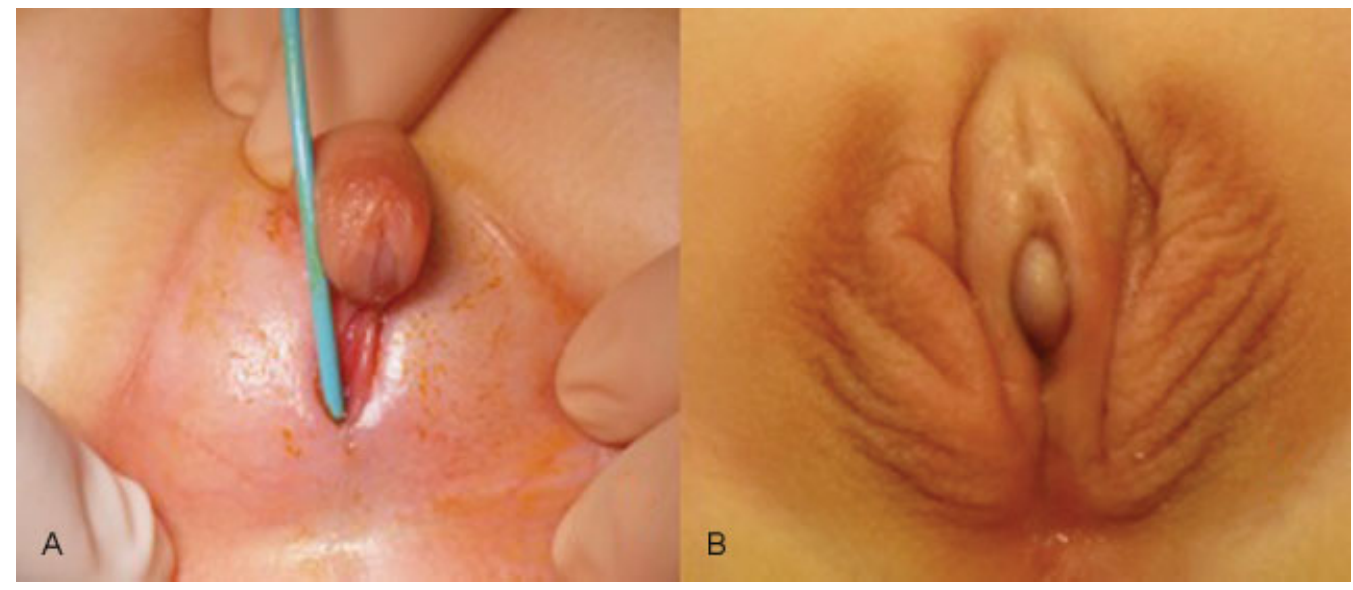

Fig. 1 Appearance of external genitalia of the patient at birth (A) and after clitoridolabioplasty (B).

\section{Case Report}

The patient was a girl ( $46 \mathrm{XX}$ ) born at 39 weeks of gestation with ambiguous genitalia. Examinations were performed at another institution where a classic form of CAH was diagnosed associated with UGS. The girl came to our attention at the age of 2 years. We performed a cystoscopy and a vaginoscopy, after having identified the presence of a $3 \mathrm{~cm}$ long UGS: we performed a clitoridolabioplasty ( - Fig. 1A, B). A year later, we decided to perform a TUM with urethrovaginoplasty and vulvoplasty. Two-step surgery was recently introduced in our center for young patients to avoid lengthy operations in the case of long UGS (genital reconstruction in the first months of life and delayed urogenital mobilization within the 1st year). We also applied this protocol in the reported case, despite the fact that the girl had only come to our attention when she was already 2 years old ( - Fig. 1 ).

\section{Surgical Technique}

A full bowel preparation and a preoperative enema were given before surgery and the patient received broad-spectrum intravenous antibiotics. The rectum was irrigated with a $0.25 \%$ neomycin solution in the operating room before the procedure and the rectum was closed with a betadinized gauze.

The initial approach was to perform an ASTRA procedure. Before the surgery, a cystoscopy and a vaginoscopy were conducted under general anesthesia ( $\sim$ Fig. 2A). We carefully evaluated the length of the CC as well as the length of the urethra and vagina and the exact position of the confluence. We placed a $3 \mathrm{~F}$ Fogarty catheter in the vagina (the Fogarty balloon was $8 \mathrm{~mm}$ in size) under vision. We then removed the cystoscope and placed an $8 \mathrm{~F}$ Foley catheter in the bladder (white probe in - Fig. 2B). The Fogarty probe helped us to identify the vagina during initial dissection and it was later replaced by a $12 \mathrm{~F}$ Foley catheter (yellow probe in - Fig. 2B). The patient was then placed in the prone position with legs abducted and a roll placed under the pubis. The surgeon stood between the patient's legs, facing the perineum. We performed a circumferential incision around the meatus extending along the midline of the perineum superiorly to our end point, which was the anterior anal border ( - Fig. 3A). The dissection started in the midline to release the UGS from the anterior rectal wall without injuring the anorectal sphincters. To achieve this, a series of $6 / 0$ polyglactin suture was put in place across the meatus of the $\mathrm{CC}$. The dissection maneuver was completed with the modification of the ASTRA technique to increase the operative field by extending the dissection up

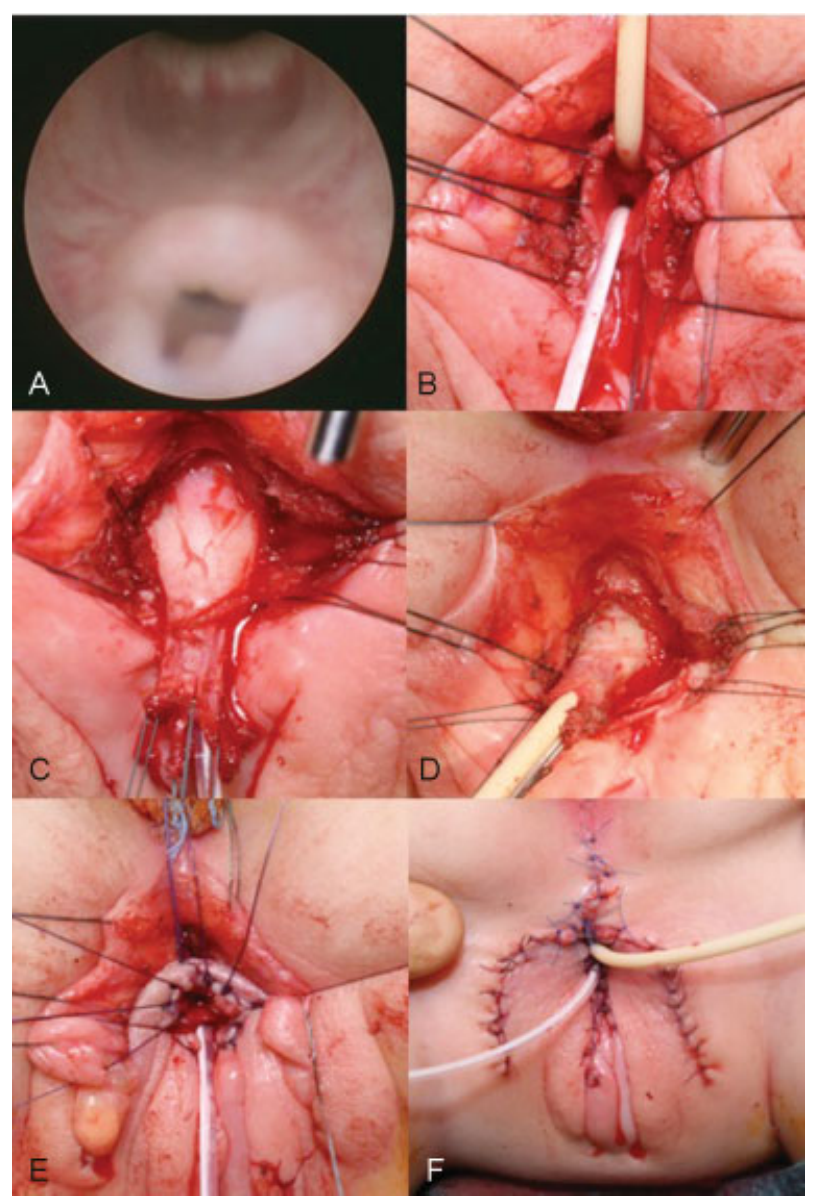

Fig. 2 Endoscopic evaluation (A); urogenital sinus dissection (B-D) with the Fogarty catheter (yellow in $B$ ) in the vagina and the Foley catheter in the urethra (white in B); once the dissection is completed urethra and vagina are sutured to the perineum (E). The vagina and vulva are then reconstructed using skin flaps (F). 


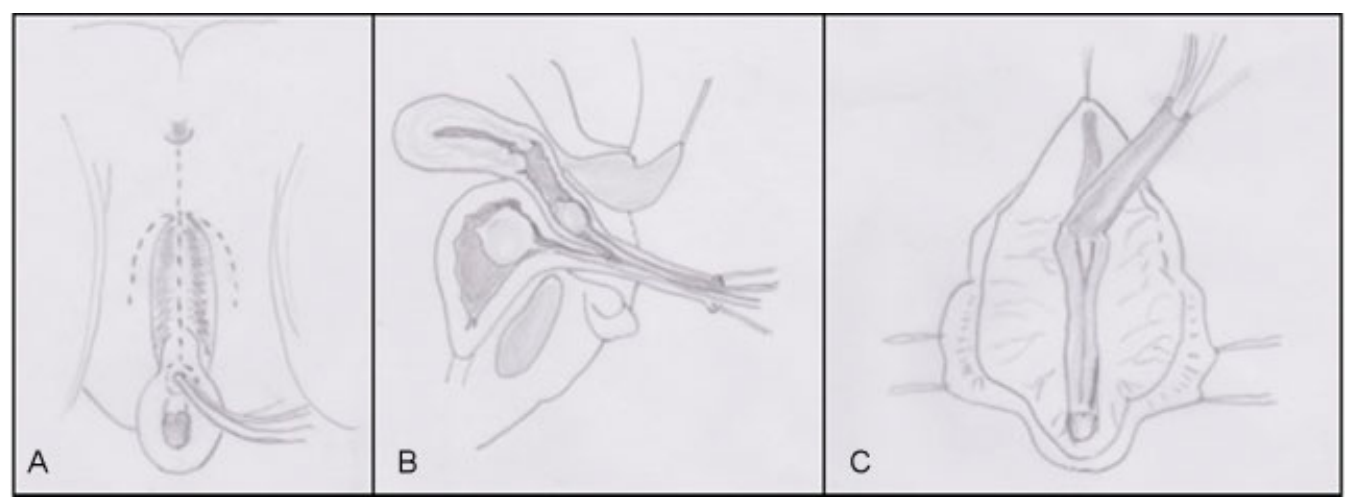

Fig. 3 Schematic picture of the surgical procedure with the patient in prone position. (A) Incision lines; (B) total urogenital sinus mobilization (sagittal view in $[B]$ and axial view in $[C]$ ).

to the anterior anorectal wall without opening it and keeping the anorectal sphincter complex intact. Following this, both the sinus and posterior vaginal walls were exposed (-Fig. 2B). The dissection proceeded circumferentially and inferiorly to release the sinus from the pubis and free the sinus (-Fig. 2C, D). Thanks to the palpable Foley catheter it was easy to see the vaginal confluence. The procedure permitted both the urinary tract and the vagina to be mobilized as one block down to the perineum, enough to achieve a good length of the vagina and a wide vaginal opening (-Fig. 3B, C). The UGS was opened in the midline: its anterior wall (which corresponds to the inferior part of the UGS with the girl in prone position, as was our case) was used to create part of the urethral introitus and to consolidate the urethra to the vulva; its posterior part was removed. The vaginal wall was then sutured to the perineal skin flaps using separate stitches of $5 / 0$ polyglactin suture ( - Fig. $2 E$ ). The midline skin incision was closed by $5 / 0$ polydioxanone suture (-Fig. $2 F$ ). The vesical Foley catheter was left in place for 5 days. After 2 weeks of the surgery, vaginal dilatations were initiated by the patient's mother once a day for the 1st week and then twice a week for a total of 3 months, increasing the size of the dilator to avoid postoperative vaginal stenosis (we started with Hegar 6 and progressed up to Hegar 9) (-Figs. 2 and 3).

We did not come up against any complications such as infection, operative wound dehiscence, stenosis, or urethral fistula in the postoperative period. The girl showed neither fecal nor urinary incontinence after being toilet trained when she stopped wearing diapers. This evaluation was based on the results of questionnaires given to the parents concerning the number of accidents in a week: soiling, social problems, urinary incontinence, ability to hold defecation, or micturition and a feeling of urgency. Furthermore, the mother was satisfied with the cosmetic results.

\section{Discussion}

UGS abnormalities are one of the most challenging problems that the pediatric urological surgeon may face. ${ }^{5}$ The anatomy is complex and varied and adequate surgical exposure can be difficult. Most patients are identified at birth with genital ambiguity, most commonly secondary to the CAH. However, it may also occur as a pure UGS anomaly with apparently normal external genitalia. ${ }^{6}$ In all cases, surgery has three main goals: (1) to separate the urinary and genital tract, (2) to correct urinary continence, and (3) to prepare for normal reproductive and sexual life through reconstruction of the vagina and external genitalia. ${ }^{7}$

In 1982, the posterior sagittal access proposed by Peña et al for patients with cloaca was quickly extended to the treatment of urogenital malformations (prostatic utricles, urethral strictures, atresia of the vagina, UGS), sectioning the anterior and posterior rectal walls in the midline or incising the muscles in the median raphe (PSTA). ${ }^{8,9}$ However, the main disadvantage of this technique was the potential damage to perirectal nerves behind the rectum. ${ }^{7}$

In 1997, Dòmini et al sectioned only the anterior rectal wall in an ASTRA to treat UGS in adrenogenital syndrome. ${ }^{10}$ This approach permitted an excellent anatomical view with a midline incision sparing the levator ani muscle complex and the external sphincter. The authors reported a low risk of fecal incontinence, easier reconstruction, and no need for a protective colostomy with proper bowel preparation.

Variations of the ASTRA, which avoid entering the rectum, have been proposed, including a prone transperineal approach (by Rink et al) and a pararectal approach. ${ }^{6,11,12}$

In the same year, Peña et al described a circumferential mobilization of the UGS as a single unit called TUM. ${ }^{13}$ This allowed a midline perineal approach without rectal dissection and with good visualization of the sinus while the patient lies supine. This procedure has gained widespread acceptance and has almost entirely replaced the older techniques. $^{3,14}$

Unfortunately, in this position the identification of the vaginal confluence is difficult, as well as the perivaginal dissection or the complete release of the vagina from the surrounding tissues. The original description of TUM limited its use to UG sinuses, less than $3 \mathrm{~cm}$ long and required discarding the excess sinus tissue $^{13,15}$ that inhibits the vagina from reaching the perineum, thereby creating a wide vaginal opening. 
Salle et al attempted a transperineal and pararectal approach in two patients. He had to convert to ASTRA in both cases to gain adequate exposure and admitted that either approach could be attempted initially, especially in infants (where tissue elasticity is excellent and planes are easier to dissect), followed by ASTRA if better visualization is needed. $^{5}$

At our center, 99 patients with type III vaginal malformations were treated from 1980 to $2014 .^{4}$

We applied the benefits of both the ASTRA and TUM techniques without splitting the rectal wall. ${ }^{16}$ We placed the patient in a prone position with bowel preparation and antibiotic therapy. It provided excellent exposure without compromising the fecal continence mechanism. As well as providing excellent exposure, we believe that this approach is safe, and leads to less morbidity than conventional ASTRA, as it avoids the splitting and suturing of the anterior rectal wall. Care was also taken to avoid injury to the anorectal sphincter muscle complex. We additionally benefited from TUM as it also avoids dissection between the urethra and vagina. As far as the choice of performing "two-step" surgery is concerned, it was related to the strong "psychological pressure" from parents, due to cultural reasons. This was the case reported; the girl came to our attention when she was already 2 years old and the parents were extremely upset because of the external aspect of the genitalia. In these cases, our experience has shown that a "two-step" approach is useful to "immediately" reduce stress for the family. Incidentally, in patients under 3 years of age, we perform the operation as a single procedure. We acknowledge that the main limitation of our article is that it is a case report and further studies are required to draw definitive conclusions. Moreover, longterm outcomes, especially during adolescence, should be assessed. As for the simplicity of the technique, we believe that the correction in older patients is more difficult, but this is a consideration which also applies to the other procedures (e.g., pure TUM, pure ASTRA).

In conclusion, the approach described above combines the advantages of ASTRA and TUM: on the one hand, it is an anterior sagittal approach that does not require rectum splitting, while on the other it has the benefit of avoiding dissection between the urethra and vagina. It is a feasible procedure, which provides excellent exposure and easy identification of the vaginal confluence for $\mathrm{CAH}$ associated with long UGS. Therefore, it may be considered a valid alternative to the standard techniques.

\section{References}

1 Rey R, Picard JY. Embryology and endocrinology of genital development. Baillieres Clin Endocrinol Metab 1998;12(1):17-33

2 Joint LWPES/ESPE CAH Working Group. Consensus statement on 21-hydroxylase deficiency from the Lawson Wilkins Pediatric Endocrine Society and the European Society for Paediatric Endocrinology. J Clin Endocrinol Metab 2002;87(9): 4048-4053

3 Ludwikowski BM, González R. The Surgical Correction of Urogenital Sinus in Patients with DSD: 15 Years after Description of Total Urogenital Mobilization in Children. Front Pediatr 2013; $1: 41$

4 Ruggeri G, Gargano T, Antonellini C, et al. Vaginal malformations: a proposed classification based on embryological, anatomical and clinical criteria and their surgical management (an analysis of 167 cases). Pediatr Surg Int 2012;28(8):797-803

5 Salle JL, Lorenzo AJ, Jesus LE, et al. Surgical treatment of high urogenital sinuses using the anterior sagittal transrectal approach: a useful strategy to optimize exposure and outcomes. J Urol 2012;187(3):1024-1031

6 Rink RC, Pope JC, Kropp BP, Smith ER Jr, Keating MA, Adams MC. Reconstruction of the high urogenital sinus: early perineal prone approach without division of the rectum. J Urol 1997;158(3 Pt 2): 1293-1297

7 Kalfa N, Liu B, Cao M, Vilella M, Hsieh M, Baskin LS. 3-dimensional neuroanatomy of the human fetal pelvis: anatomical support for partial urogenital mobilization in the treatment of urogenital sinus. J Urol 2008;180(4, Suppl):1709-1714, discussion 1714-1715

8 Peña A, Devries PA. Posterior sagittal anorectoplasty: important technical considerations and new applications. J Pediatr Surg 1982;17(6):796-811

9 Peña A, Filmer B, Bonilla E, Mendez M, Stolar C. Transanorectal approach for the treatment of urogenital sinus: preliminary report. J Pediatr Surg 1992;27(6):681-685

10 Dòmini R, Rossi F, Ceccarelli PL, De Castro R. Anterior sagittal transanorectal approach to the urogenital sinus in adrenogenital syndrome: preliminary report. J Pediatr Surg 1997;32(5):714-716

11 Kuhn EJ, Skoog SJ, Nicely ER. The posterior sagittal pararectal approach to posterior urethral anomalies. J Urol 1994;151(5): 1365-1367

12 Pintér AB, Hock A, Vástyán A, Farkas A. Does the posterior sagittal approach with perirectal dissection impair fecal continence in a normal rectum? J Pediatr Surg 1996;31(10):1349-1353

13 Peña A. Total urogenital mobilization-an easier way to repair cloacas. J Pediatr Surg 1997;32(2):263-267, discussion 267-268

14 Rink RC, Metcalfe PD, Cain MP, Meldrum KK, Kaefer MA, Casale AJ. Use of the mobilized sinus with total urogenital mobilization. J Urol 2006;176(5):2205-2211

15 Peña A, Levitt MA, Hong A, Midulla P. Surgical management of cloacal malformations: a review of 339 patients. J Pediatr Surg 2004;39(3):470-479, discussion 470-479

16 Leite MT, Fachin CG, de Albuquerque Maranhão RF, Shida ME, Martins JL. Anterior sagittal approach without splitting the rectal wall. Int J Surg Case Rep 2013;4(8):723-726 